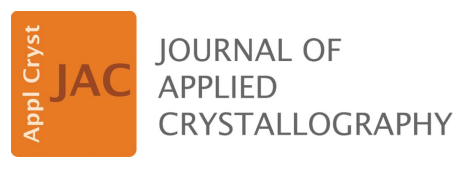

ISSN 1600-5767

Received 12 April 2015

Accepted 20 September 2015

Edited by A. Borbély, Ecole National Supérieure des Mines, Saint-Etienne, France

Keywords: three-dimensional electron backscatter diffraction; serial sectioning; grain boundary distribution; alignment; kernel density estimation.

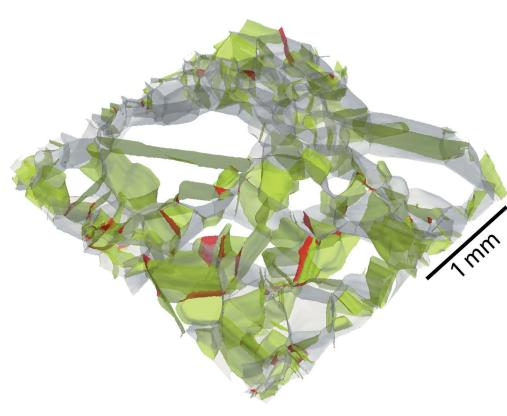

C 2015 International Union of Crystallography

\section{Three-dimensional characterization of grain boundaries in pure nickel by serial sectioning via mechanical polishing}

\author{
Hadi Pirgazi, ${ }^{a *}$ Krzysztof Glowinski, $^{\mathrm{b}}$ Adam Morawiec ${ }^{\mathrm{b}}$ and Leo A. I. Kestens ${ }^{\mathrm{a}, \mathrm{c}}$ \\ a Department of Materials Science and Engineering, Ghent University, Technologiepark 903, Ghent, 9052, Belgium, \\ b Institute of Metallurgy and Materials Science, Polish Academy of Sciences, Reymonta 25, Krakow, 30-059, Poland, and \\ ${ }^{\mathbf{c}}$ Department of Materials Science and Engineering, Delft University of Technology, Mekelweg 2, Delft, 2628CD, The \\ Netherlands. *Correspondence e-mail: hadi.pirgazi@ugent.be
}

Five macroscopic boundary parameters can be extracted from three-dimensional orientation maps. Serial sectioning, which includes consecutive steps of material removal, and electron backscatter diffraction (EBSD) measurement were employed to extract a stack of two-dimensional sections of a pure nickel sample. The EBSD patterns were collected from large millimetre scale areas and mechanical polishing was applied to prepare the sections. The three-dimensional microstructure was then reconstructed from these sections. A new alignment algorithm based on the minimization of misorientation between two adjacent sections has been developed to accurately align the sections. Differently from the conventional alignment methods, the new algorithm corrects not only the translational misalignment but also rotational and plane parallelity misalignments. The aligned three-dimensional microstructure exhibits smooth grain boundary planes and continuous orientation gradients inside the grains as experimental scatter induced by misalignment was largely removed. Grain boundaries were reconstructed from the aligned three-dimensional map, and the distribution of boundaries in the domain of five macroscopic boundary parameters was computed using kernel density estimation. Methods for estimating the reliability of the distributions are demonstrated. This distribution is compared with the distributions obtained previously for other face-centered cubic materials, including a different pure nickel sample.

\section{Introduction}

Although many microstructural features of materials can be characterized with conventional two-dimensional analytical techniques, a number of important microstructural properties (e.g. detailed grain boundary features) can only be measured in three dimensions. Five so-called macroscopic parameters are required to describe a grain boundary. Three parameters are associated with the crystallographic misorientation across the grain boundary. The other two parameters specify the boundary plane normal, also known as the boundary plane inclination (Randle, 1996, 2010). Many important material properties such as corrosion resistance (Randle, 2010), brittleness and toughness (Watanabe \& Tsurekawa, 2004) are affected by the structure of grain boundaries. The procedure of improving material properties by changing the properties of grain boundaries is known as grain boundary engineering (GBE) (Randle, 2010). For GBE it is necessary to be able to derive, represent and investigate the five-parameter grain boundary distribution, which represents the area frequency of boundaries with a specific type of misorientation and boundary plane normal (Saylor et al., 2004). The five-parameter distribution can be obtained using stereological 
methods (Rohrer et al., 2010) based on four parameters determined from two-dimensional sections. However, application of these methods to textured materials is limited. Therefore, it is more convenient to employ three-dimensional characterization techniques, which allow for the direct assessment of all five parameters. To obtain a reliable distribution of the grain boundary parameters, it is important to collect the crystallographic information from large numbers of grains. Three-dimensional images of microstructures containing many grains are commonly obtained using either high-energy X-rays (Jensen \& Poulsen, 2012) or serial sectioning combined with electron backscatter diffraction (EBSD) (DeHoff, 1983; Zaefferer et al., 2008; Uchic et al., 2012).

Serial sectioning has been widely used to acquire threedimensional data at the macro- and microscale of opaque materials, e.g. to derive the distribution of the grain boundary parameters for magnesia (Saylor et al., 2003), lead-tin alloy (Rowenhorst \& Voorhees, 2012), yttria (Dillon \& Rohrer, 2009), nickel (Li et al., 2009; Rohrer et al., 2010) and CuZr alloy (Khorashadizadeh et al., 2011). In this technique, a series of closely spaced parallel sections reveal the third dimension of the microstructure. The conventional method for serial sectioning involves the cyclic removal of parallel layers of the sample, followed by imaging (e.g. by EBSD) of the planar sections (DeHoff, 1983; Alkemper \& Voorhees, 2001). The removal of the material for serial sectioning can be performed with different methods: e.g. by mechanical polishing, electropolishing, focused ion beam (FIB) removal and femtosecond laser ablation (Echlin et al., 2011). Regardless of the method employed for the preparation of the sections, the serial sectioning technique suffers from the misalignment between consecutive sections, which needs to be accurately corrected before further data processing (Uchic, 2011).

The goal of this work is to develop a new alignment procedure to correct any spatial and orientational misalignment between the two-dimensional EBSD sections prepared by mechanical polishing. The new procedure was applied to a stack of consecutive EBSD maps acquired from a sectioned pure nickel sample. The reconstructed microstructure was used to extract the grain boundary network and to compute the corresponding boundary distribution.

\section{Experimental procedures}

\subsection{Material and three-dimensional characterization}

Polycrystalline pure nickel sheet with $1.0 \mathrm{~mm}$ thickness was used for this study. The sample was annealed for $1 \mathrm{~h}$ in the open atmosphere at a temperature of $1273 \mathrm{~K}$, which resulted in a boundaries). bimodal grain size distribution with large grains (with grain sizes of $0.3-0.9 \mathrm{~mm}$ ) surrounded by small grains (with an average size of $200 \mu \mathrm{m}$ ). Twins appeared in the annealed microstructure, and the grain boundary analysis revealed that about $41 \%$ of the area of all boundaries exhibits $\Sigma 3(60 \%$ [111]) misorientation. Another type of coincident-site lattice boundary that is present in the microstructure is the $\Sigma 9\left(38.9^{\circ}\right.$ \% [110]) boundary, with an area fraction of $1.5 \%$. The fractions were estimated assuming a misorientation tolerance of $3^{\circ}$.

Wide-field three-dimensional EBSD was performed on the $\mathrm{RD}-\mathrm{TD}$ section (RD is the rolling direction and TD the transverse direction) of the sample by the serial sectioning technique. This method involves consecutive steps of sample preparation and EBSD measurement. The sample preparation was done by conventional mechanical polishing to remove a layer with a thickness of $7 \mu \mathrm{m}$. After each polishing step, the EBSD measurement was performed in a high-resolution field emission gun scanning electron microscope (of type FEI QUANTA 450) on a large area $(3200 \times 3200 \mu \mathrm{m})$ with an inplane step size of $6 \mu \mathrm{m}$ to obtain the local crystallographic orientations. EBSD patterns were collected by means of the $T S L$-OIM software (EDAX Inc., Mahwah, NJ, USA). In order to properly align scan areas on subsequent layers, microVickers indentations were used as fiducial marks on the sample. These indentations were also used to measure the thickness of each removed layer. In total 50 sections were prepared, to cover a total depth of $350 \mu \mathrm{m}$.

\subsection{Section alignment}

In spite of the meticulous mechanical alignment of the specimen by the micro-Vickers indentations, the maps still may be slightly misaligned. Different methods have been proposed to further optimize the alignment of the serial sectioning data. One generally accepted approach was proposed by Lee et al. (2007), which is a cross correlation

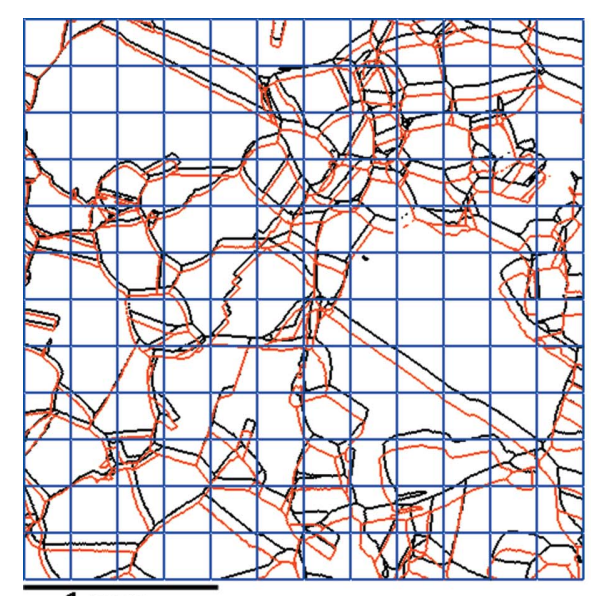

$1 \mathrm{~mm}$

\section{Figure 1}

(a)

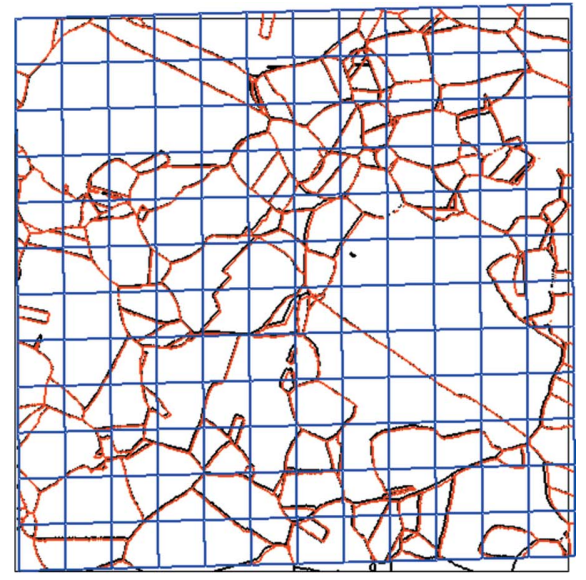

(b)

Grain boundaries of two consecutive sections: $(a)$ before and $(b)$ after spatial alignment. The section with the black boundaries is kept fixed, and the other is moved to obtain the best alignment. The blue grid represents the distortion correction that has been applied on the moving section (with red grain 
procedure based on the minimization of the average misorientation between two adjacent sections. Serial sectioning data collected by a mechanical polishing technique may generally suffer from translational, rotational and stretching misalignment (cf. Fig. 1a). The latter occurs when two consecutive sections are not entirely parallel, which causes dimensional distortion between adjacent sections.

A two-step registration algorithm (which will be referred to as the full alignment) is proposed here to obtain the best possible alignment between the two-dimensional sections. The first step is to correct for any spatial translation, rotation and distortion between the layers. In this step, the lower section is kept fixed, but the four corners of the upper section are freely moved in the plane of that section to shift, rotate, stretch or shrink the map. It is assumed that the distortion in the map is linear and thus the new coordinates of the scan points are calculated from the coordinates of the corners by applying a bilinear interpolation. The calculated coordinates are then rounded to multiples of step size value. For each position of the four corners, the average misorientation angle between two sections was calculated by averaging out the misorientation angle between corresponding pixels in the upper and lower sections. Finally, the position of the four corners that resulted in the minimum average misorientation between two adjacent sections is selected as the optimum for the best spatial alignment ( $c f$. Fig. 1b).

The second step of the alignment algorithm is to correct the misorientation between sections resulting from in-plane rotational misalignment as well as out-of-plane (plane parallelity) misalignment. This type of misalignment is apparent from the difference in inverse pole figure coded colors of the same grains in two consecutive sections ( $c f$. Fig. $2 a$ and $2 b$ ). To this purpose, a transformation matrix was composed from individual transformations around RD, TD and ND (normal direction):

$$
\mathbf{T}=\mathbf{T}_{\mathrm{RD}}\left(\theta_{\mathrm{RD}}\right) \mathbf{T}_{\mathrm{TD}}\left(\theta_{\mathrm{TD}}\right) \mathbf{T}_{\mathrm{ND}}\left(\theta_{\mathrm{ND}}\right),
$$

where $\mathbf{T}_{\mathrm{RD}}\left(\theta_{\mathrm{RD}}\right)$ is a transformation of $\theta_{\mathrm{RD}}$ around the RD axis [and equally for $\mathbf{T}_{\mathrm{TD}}\left(\theta_{\mathrm{TD}}\right)$ and $\mathbf{T}_{\mathrm{ND}}\left(\theta_{\mathrm{ND}}\right)$ ]. The angles $\theta_{\mathrm{RD}}$, $\theta_{\mathrm{TD}}$ and $\theta_{\mathrm{ND}}$ were changed between $-5^{\circ}$ and $5^{\circ}$ with a $0.1^{\circ}$ step. For all points in the upper EBSD scan, the orientations were transformed by the $\mathbf{T}$ matrix and the average misorientation angle between the two sections was calculated. Finally, the transformation matrix that resulted in the minimum average misorientation between two adjacent sections was chosen to correct the misorientation caused by the misalignment. The two steps of the alignment algorithm were performed repeatedly until no further correction was required. Fig. 2 shows the results of the proposed alignment procedure. It can be clearly seen that misalignment (translational, rotational and stretching) has been reduced ( $c f$. Fig. $2 c$ ). Note that the corresponding grains of two different sections after the two-step alignment ( $c f$. Figs. $2 a$ and $2 c$ ) represent the same orientation (color).

\section{Results and discussion}

\subsection{Aligned three-dimensional microstructure}

Fig. 3 shows the three-dimensional volume of the reconstructed microstructure before alignment, after translational alignment [by shifting one section along RD and TD with respect to the other section, as proposed by Lee et al. (2007)] and after full alignment (as employed in this study). In the original results (i.e. before alignment; $c f$. Fig. $3 a$ ), there are discontinuities in grain orientations while the boundary planes exhibit a distorted ledged character. The translational alignment (cf. Fig. $3 b$ ) makes grain boundaries smoother, but abrupt changes of orientation between sections remain. The full alignment ( $c f$. Figs. $3 c$ and $3 d$ ) not only improves the boundary curvatures but also leads to more continuous orientation gradients in the grains. This observation was quantitatively analyzed by computing the average misorientation between the corresponding pixels of the adjacent sections. The results indicate that the full alignment reduces this misorientation from $10.0^{\circ}$ (for the original results) and $5.8^{\circ}$ (after translational alignment) to $2.8^{\circ}$.

\subsection{Five-parameter grain boundary distribution}

The network of grain boundaries contained in the microstructure was extracted from the aligned three-dimensional

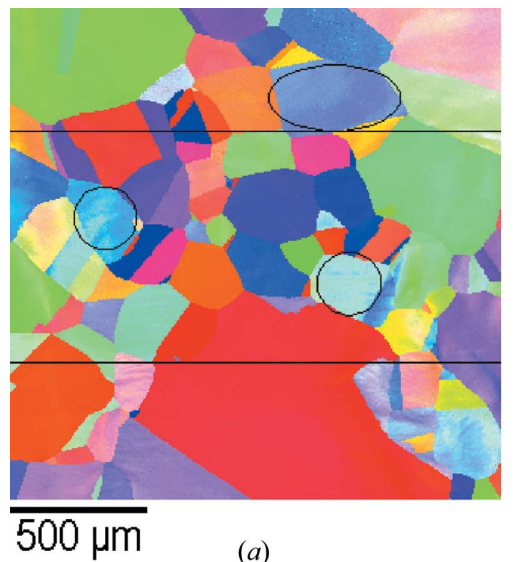

Figure 2

Results of the full registration of two consecutive sections: $(a)$ the first section, which is considered as the fixed section, $(b)$ the second section before alignment and $(c)$ the second section after full alignment. The black lines and circles can be used for comparison between the results. 


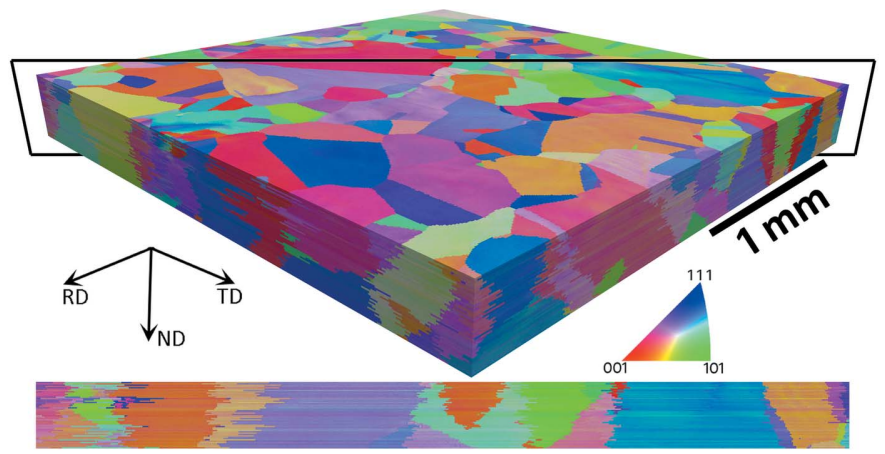

(a)

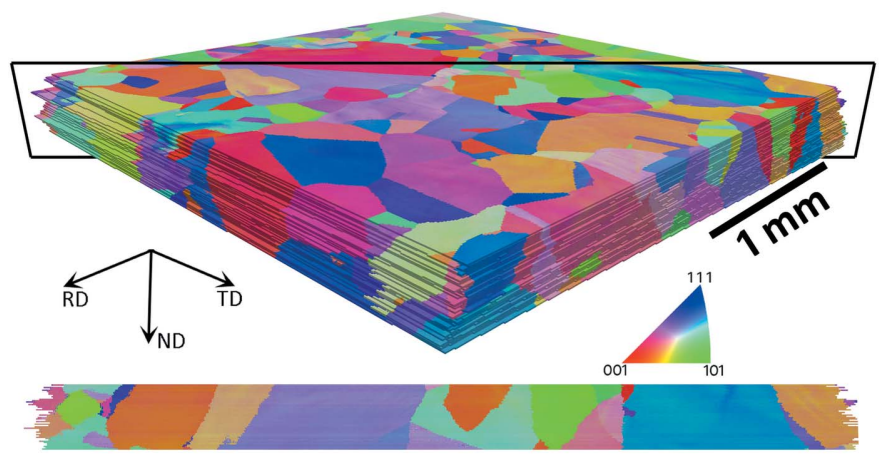

(c)

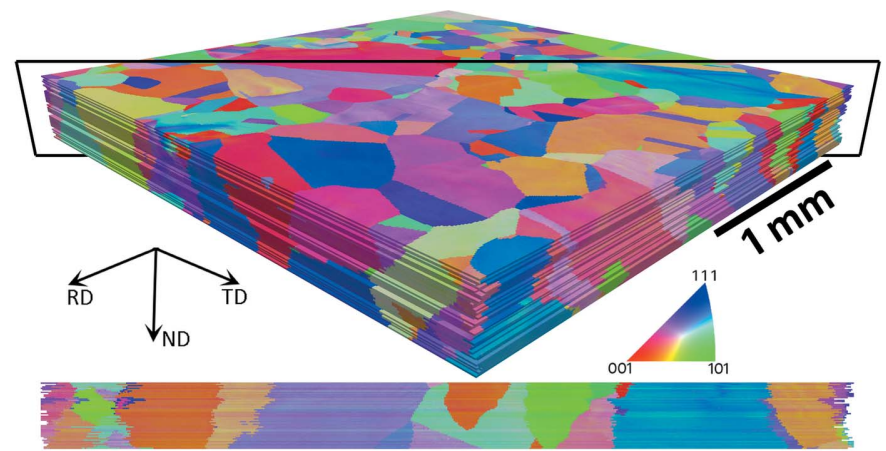

(b)

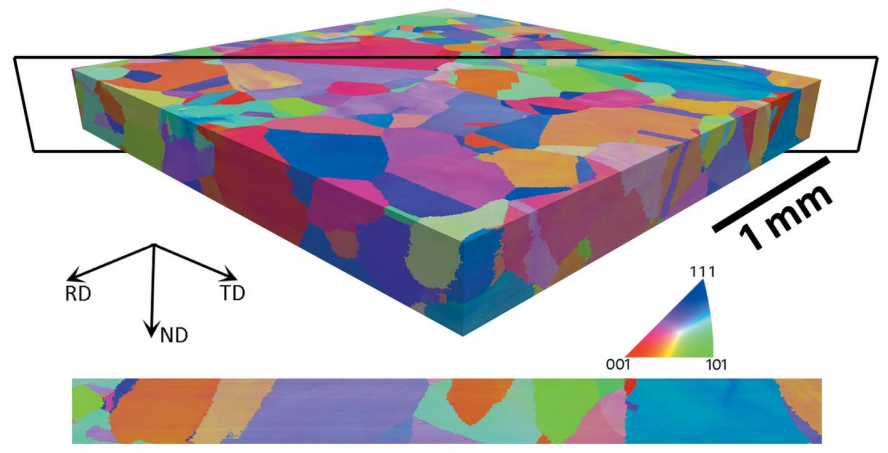

(d)

Figure 3

ND inverse pole figure volume map of the reconstructed microstructure together with the cross section corresponding to the specified plane (black lines): (a) the stack of the original EBSD data without any alignment, $(b)$ after translational alignment, $(c)$ after full alignment, and $(d)$ after full alignment and cropping of the lateral sides of the volume. The size of this volume is $3000 \times 3000 \times 350 \mu \mathrm{m}$, and it contains 470 grains.

orientation map using the DREAM.3D program (Groeber \& Jackson, 2014). The geometric surfaces of boundaries were reconstructed in the form of a mesh of triangular segments with misorientations of neighboring grains and local boundary normals associated with each segment. The network consisted of about 2100 distinct boundaries (cf. Fig. 4) and it was modeled by approximately $2.5 \times 10^{6}$ segments. These data were used to calculate the function representing the distribution of grain boundaries in the space of macroscopic parameters. Following the common way of presenting boundary distributions (Saylor et al., 2003), the function is plotted in the form of sections for fixed misorientations and varying boundary normals displayed in stereographic projection. The populations are expressed as multiples of random distribution (MRD).

The obtained distribution complements the already large collection of grain boundary distributions determined for cubic materials (see e.g. Dillon \& Rohrer, 2009; Li et al., 2009; Glowinski \& Morawiec, 2014; Beladi et al., 2014; Saylor et al., 2003). A considerable part of this collection is related to facecentered cubic (f.c.c.) metals, including, for example, Ni-based superalloy IN100 (Glowinski \& Morawiec, 2014) and austenite (Beladi et al., 2014). Our result needs to be directly compared with that for pure fine-grained nickel determined by Li et al. (2009) using FIB-based sectioning. Li's material differed from that investigated in our study by processing history and, in consequence, by average grain size and grain size distribution:
Li's sample displayed a unimodal distribution with the mean of $11 \mu \mathrm{m}$.

Although a large volume of the microstructure was analyzed in our experiment (almost five orders of magnitude larger than those investigated in FIB-based experiments), the relatively large grain diameters implied that the overall

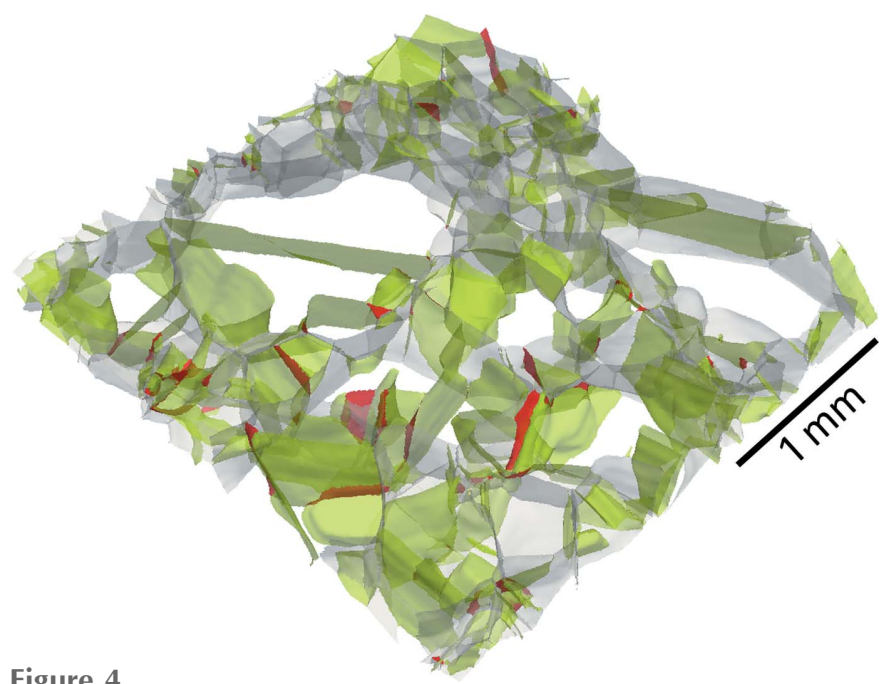

Figure 4

Network of grain boundaries reconstructed from three-dimensional EBSD data of the studied nickel sample. Boundaries with the $\Sigma 3$ and $\Sigma 9$ misorientations (with a tolerance of $3^{\circ}$ ) are colored in green and red, respectively; all other boundaries are shaded. 
number of grains contained in this volume was relatively small; it was about half of that investigated by Li et al. (2009). The total area of boundaries in the studied sample was $2.7 \times$ $10^{7} \mu \mathrm{m}^{2}$; for comparison, it was only $1.4 \times 10^{4} \mu \mathrm{m}^{2}$ in one (out of five) of the specimens of Li et al. (2009). However, the error of the boundary distribution is affected by the number of individual boundaries not the boundary area, and therefore, the investigated data set is statistically smaller than that considered by Li et al. (2009). In order to deal with such poor statistics, the most accurate and reliable methods for estimating grain boundary distributions must be used.

Most of the boundary distributions published so far, including that reported by Li et al. (2009), were obtained using a method based on dividing the five-dimensional space of boundary parameters into equi-volume bins and summing areas of mesh segments falling into a specific bin (Saylor et al., 2003). However, several deficiencies of that method were recently pointed out (Glowinski \& Morawiec, 2014). Briefly, elongated shapes of some bins and lack of correspondence between bin dimensions and experimental resolutions lead to artifacts that hinder analysis of the distributions.

These artifacts are significantly reduced, and the quality of the distributions is improved, when the binning is abandoned in favor of the kernel density estimation (KDE) (Glowinski \& Morawiec, 2014). With KDE, the distribution is probed in points uniformly dispersed in the boundary space, and areas of boundaries that are no farther (in terms of a metric defined in that space) from a given point than an assumed limiting radius are summed. Since the experimental accuracies of determination of grain misorientations and boundary plane inclinations are considerably different, it is beneficial to take advantage of the fact that the grain boundary space is a Cartesian product of the grain-misorientation and boundarynormal subspaces (Morawiec, 2009), and to use metrics in each of the two subspaces and two different limiting radii somehow linked to the experimental resolution and the number of investigated boundaries. Thus, the first step in our KDE procedure is to identify boundary segments with misorientations no farther than a certain radius $\rho_{\mathrm{m}}$ from that fixed misorientation. Then, the grain boundary distribution at this misorientation is probed at densely dispersed boundary normals. To get a value at a given normal, areas of the identified segments that have normals deviating from this normal by less than a limiting radius $\rho_{\mathrm{p}}$ are summed. The final grain boundary distribution is obtained from these values by normalization.

Since the $\Sigma 3$ and $\Sigma 9$ misorientations occur frequently in the microstructure ( $c f$. Fig. 4), the corresponding sections through the boundary distribution are expected to be the most reliable. The $\Sigma 3$ and $\Sigma 9$ sections are presented in Figs. 5(a) and 5(b). They were obtained using the KDE-based approach. The metrics and limiting radii $\left(\rho_{\mathrm{m}}=3^{\circ}, \rho_{\mathrm{p}}=7^{\circ}\right)$ were the same as those used by Glowinski \& Morawiec (2014). In brief, the distance in the misorientation subspace is given by the minimum angle of a rotation transforming one misorientation into another; the minimization is over symmetrically equivalent boundary representations. The distance in the boundary- normal subspace is a function of the angles between vectors normal to boundaries.

In the $\Sigma 3$ section, there is a strong peak with an intensity of $2480 \mathrm{MRD}$ at the (111) pole. Besides the coherent twin

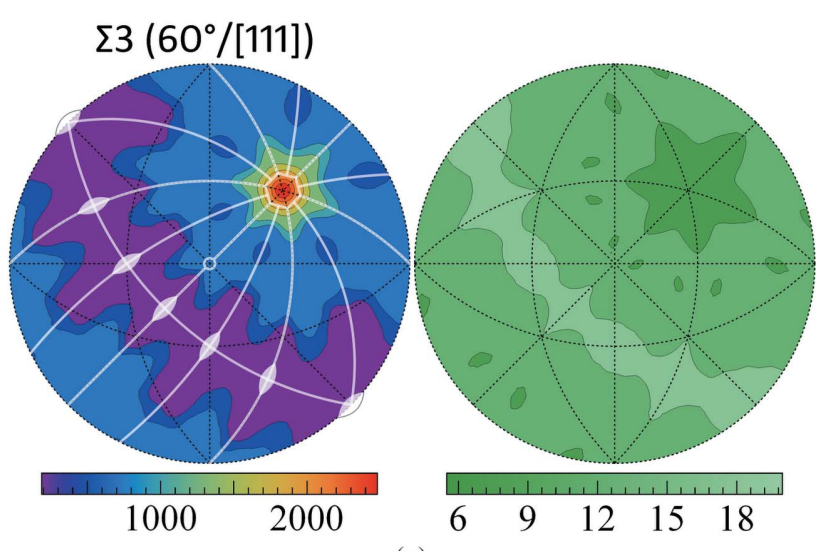

(a)
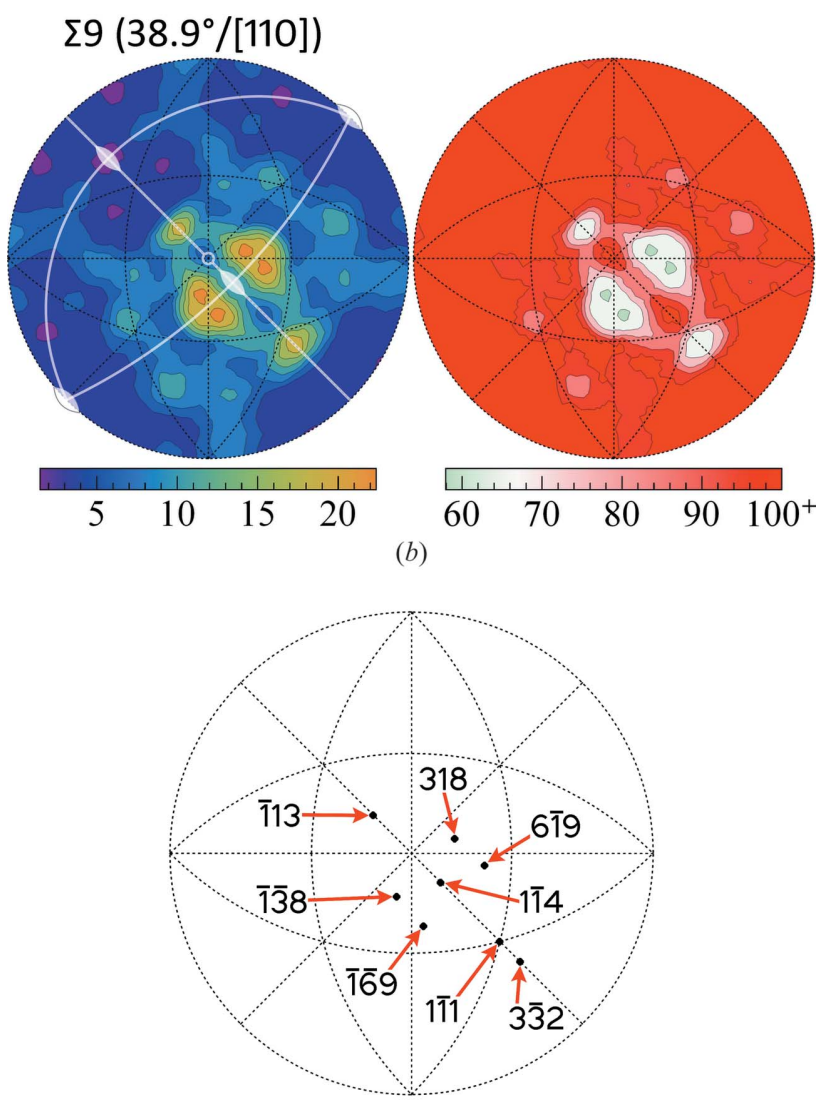

(c)

Figure 5

Sections of the grain boundary distribution for the (a) $\Sigma 3$ and (b) $\Sigma 9$ misorientations; in each pair the left-hand plot represents the frequency of occurrence of boundary planes for a given misorientation, while the right-hand plot represents the corresponding relative error. Contours of the distributions are given in MRD, whereas isolines for the errors are expressed in percent. Isolines for errors exceeding $100 \%$ are not plotted; ' $100+$ ' means 100 and more. White symbols denote symmetry elements of the sections: lines correspond to mirror reflections, circles stand for inversion centers, while two- and sixfold symmetry axes are represented by ovals and a hexagon, respectively. Relevant poles for the $\Sigma 9$ section are indicated in $(c)$. 
boundaries, also incoherent $\Sigma 3$ boundaries with boundary planes other than (111) exhibit frequencies significantly exceeding the random average. The minimum value in the $\Sigma 3$ section is about 200 MRD. These numbers are quite reliable as their relative errors vary between 6 and $20 \%$; here and below, the relative error of a distribution at a given point is estimated as $\varepsilon=1 /(f n v)^{1 / 2}$, where $f$ denotes the value of the distribution, $n$ is the number of distinct grain boundaries in the network, and $v$ stands for the volume determined by $\rho_{\mathrm{m}}$ and $\rho_{\mathrm{p}}$ (Glowinski \& Morawiec, 2014). The $\Sigma 3$ section in Fig. 5(a) very much resembles functions obtained for other f.c.c. metals having significant fractions of $\Sigma 3$ boundaries. This applies to fine-grained pure nickel with a unimodal grain size distribution (Li et al., 2009), and also to superalloy IN100 (Glowinski \& Morawiec, 2014) and austenite (Beladi et al., 2014).

Interestingly, the other relatively reliable section of the grain boundary distribution, i.e. $\Sigma 9$, is considerably different from those observed in other f.c.c. metals. There is a peak with an intensity of $22 \pm 13$ MRD near the (318) pole [with the boundary plane in the second grain being (169); see Fig. $5 b]$. Because of symmetries of the boundary distributions, the peak is reflected three times ( $c f$. Patala \& Schuh, 2013), and it is seen as two pairs of partly overlapping peaks. These four peaks represent the same physical boundaries. Besides that, there is a peak near the (33) pole and its equivalent at ( $\overline{1} 13)$, both with heights of $18 \pm 11 \mathrm{MRD}$; these maxima represent tilt boundaries. The respective planes in the second crystal are $(\overline{1} 1 \overline{3})$ and $(3 \overline{32})$.

For comparison, regardless of the computational method, a common feature of the distributions obtained previously for the f.c.c. metals was a peak near the $\Sigma 9 /(1 \overline{1} 4)$ symmetric tilt boundary (Beladi et al., 2014; Li et al., 2009). The (318) pole apparent in our distribution is located about $21^{\circ}$ from (114); it is rather unlikely that this shift is caused by experimental and reconstruction errors. However, the shape of our distribution function may be affected by fluctuations resulting from poor statistics. In the case of small data sets, quite high peaks may represent single large-area boundaries. Although this is not true in the case of our $\Sigma 9$ section as it was verified that there are contributions from about 40 distinct $\Sigma 9$ boundaries, this number is still too small to draw any conclusions. Besides the (114) peak, a maximum in the neighborhood of the (1111) pole (corresponding to a tilt boundary) was also reported by Li et al. (2009) and Glowinski \& Morawiec (2014). The difference in

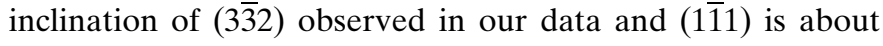
$10^{\circ}$; this value exceeds, but only slightly, the experimental resolution for boundary plane parameters.

It is also interesting to address the question asked by Li $e t$ al. (2009), whether $\Sigma 9$ boundaries are numerous because of their low energy or because of a large number of intersecting $\Sigma 3$ boundaries. It is well known that, if two $\Sigma 3$ boundaries meet at the triple junction, the third boundary at this junction has the $\Sigma 9$ misorientation (Gertsman, 2001). It turns out that all $\Sigma 9$ boundaries in the investigated volume are adjacent to $\Sigma 3$ boundaries ( $c f$. Fig. 4), i.e. in the considered case, the presence of $\Sigma 9$ boundaries is caused by the high population of $\Sigma 3$ boundaries.
It makes sense to ask why particular boundary planes are preferred among these $\Sigma 9$ boundaries. Molecular dynamics simulations indicate that $\Sigma 9$ tilt boundaries with planes in the [110] zone have lower energies compared to other $\Sigma 9$ boundaries, and the minimum energy is located at the (1114)

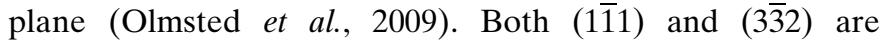
contained in this zone. The currently available data do not indicate other local energy minima. In particular, there is no explanation for the peak at (318).

For nickel with a unimodal grain size distribution, also $\Sigma 5$ and $\Sigma 7$ sections were analyzed by Li et al. (2009). The values of the distribution were low (in the ranges of 0.3-0.9 MRD and $0.57-0.81 \mathrm{MRD}$, respectively). Using the KDE-based approach, we computed the $\Sigma 5$ and $\Sigma 7$ sections for the specimen studied in this work and, for comparison, for a subset of the data that were analyzed by Li et al. (2009). The obtained results differ significantly between these two samples [and none of them are similar to the original distribution presented by Li et al. (2009)]. However, for both specimens, the accompanying errors exceed $100 \%$. Therefore, there are no grounds for drawing any conclusions on $\Sigma 5$ and $\Sigma 7$ boundaries in these two Ni samples. This statement is also true for the original $\Sigma 5$ and $\Sigma 7$ sections given by Li et al. (2009).

\section{Conclusions}

Serial sectioning by the mechanical polishing technique was used to collect 50 planar EBSD sections from a pure nickel sample. A two-step alignment algorithm was proposed to correct any misalignment, including translation, rotation and distortion as well as the misorientation between the adjacent sections. The results showed that this full alignment procedure produces a more reliable grain boundary plane morphology and continuous orientation gradients inside the grains. The latter could not be achieved with the conventional alignment technique, which only includes translational correction.

From the fully aligned data, the grain boundary network was reconstructed, and the five-parameter boundary distribution was computed using the recently developed approach based on kernel density estimation. The reliability of this distribution was evaluated. This included calculation of its errors and verification of the number of boundaries contributing to selected peaks. Our distribution obtained for the investigated $\mathrm{Ni}$ sample, which has large grain sizes and a bimodal grain size distribution, was compared with the distribution computed for fine-grained nickel with a unimodal grain size distribution (Li et al., 2009). The corresponding $\Sigma 3$ sections were similar, while there were some differences in the $\Sigma 9$ sections. For the Ni with a unimodal grain size distribution, peaks were observed at the (114) and (111) planes, while for

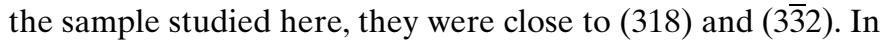
other parts of both distributions, either the errors exceed $100 \%$ or the maxima are random fluctuations resulting from individual large-area boundaries. In the studied material, all $\Sigma 9$ boundaries appear at intersections of two $\Sigma 3$ boundaries, 
which indicates that $\Sigma 9$ boundaries were produced by triple junction coalescence of $\Sigma 3$ boundaries.

\section{Acknowledgements}

This research was financially supported by the Belgian Science Foundation (FWO) under contact No. G.0257.14N. KG received funds for his work from the National Science Center Poland based on decision DEC-2014/12/T/ST8/00086.

\section{References}

Alkemper, J. \& Voorhees, P. W. (2001). J. Microsc. 201, 388-394. Beladi, H., Nuhfer, N. T. \& Rohrer, G. S. (2014). Acta Mater. 70, 281289.

DeHoff, R. T. (1983). J. Microsc. 131, 259-263.

Dillon, S. J. \& Rohrer, G. S. (2009). J. Am. Ceram. Soc. 92, 1580-1585.

Echlin, M. P., Husseini, N. S., Nees, J. A. \& Pollock, T. M. (2011). Adv. Mater. 23, 2339-2342.

Gertsman, V. Y. (2001). Acta Cryst. A57, 369-377.

Glowinski, K. \& Morawiec, A. (2014). Met. Mater. Trans. A, 45, 31893194.

Groeber, M. \& Jackson, M. (2014). Integr. Mater. Manuf. Innov. 3, 5. Jensen, D. J. \& Poulsen, H. F. (2012). Mater. Charact. 72, 1-7.

Khorashadizadeh, A., Raabe, D., Zaefferer, S., Rohrer, G. S., Rollett, A. D. \& Winning, M. (2011). Adv. Eng. Mater. 13, 237-244.

Lee, S. B., Rollett, A. D. \& Rohrer, G. S. (2007). Recrystallization and Grain Growth III, Parts 1 and 2, edited by S. J. L. Kang, M. Y. Huh,
N. M. Hwang, H. Homma, K. Ushioda \& Y. Ikuhara, pp. 915-920. Zurich: Trans Tech Publications.

Li, J., Dillon, S. J. \& Rohrer, G. S. (2009). Acta Mater. 57, 43044311.

Morawiec, A. (2009). J. Appl. Cryst. 42, 783-792.

Olmsted, D. L., Foiles, S. M. \& Holm, E. A. (2009). Acta Mater. 57, 3694-3703.

Patala, S. \& Schuh, C. A. (2013). Philos. Mag. 93, 524-573.

Randle, V. (1996). The Role of the Coincidence Site Lattice in Grain Boundary Engineering. London: Institute of Materials.

Randle, V. (2010). Mater. Sci. Technol. 26, 253-261.

Rohrer, G. S., Li, J., Lee, S., Rollett, A. D., Groeber, M. \& Uchic, M. D. (2010). Mater. Sci. Technol. 26, 661-669.

Rowenhorst, D. J. \& Voorhees, P. W. (2012). Annu. Rev. Mater. Res. 42, 105-124.

Saylor, D. M., El-Dasher, B. S., Adams, B. L. \& Rohrer, G. S. (2004). Met. Mater. Trans. A, 35, 1981-1989.

Saylor, D. M., Morawiec, A. \& Rohrer, G. S. (2003). Acta Mater. 51, 3663-3674.

Uchic, M. D. (2011). Computational Methods for MicrostructureProperty Relationships, edited by S. Ghosh \& D. Dimiduk, pp. 3152. New York: Springer US.

Uchic, M., Groeber, M., Shah, M., Callahan, P., Shiveley, A., Scott, M., Chapman, M. \& Spowart, J. (2012). First International Conference on 3D Materials Science, edited by M. De Graef, H. F. Poulsen, A. Lewis, J. Simmons \& G. Spanos. Hoboken: John Wiley \& Sons.

Watanabe, T. \& Tsurekawa, S. (2004). Mater. Sci. Eng. A, 387-389, 447-455.

Zaefferer, S., Wright, S. I. \& Raabe, D. (2008). Met. Mater. Trans. A, 39, 374-389. 